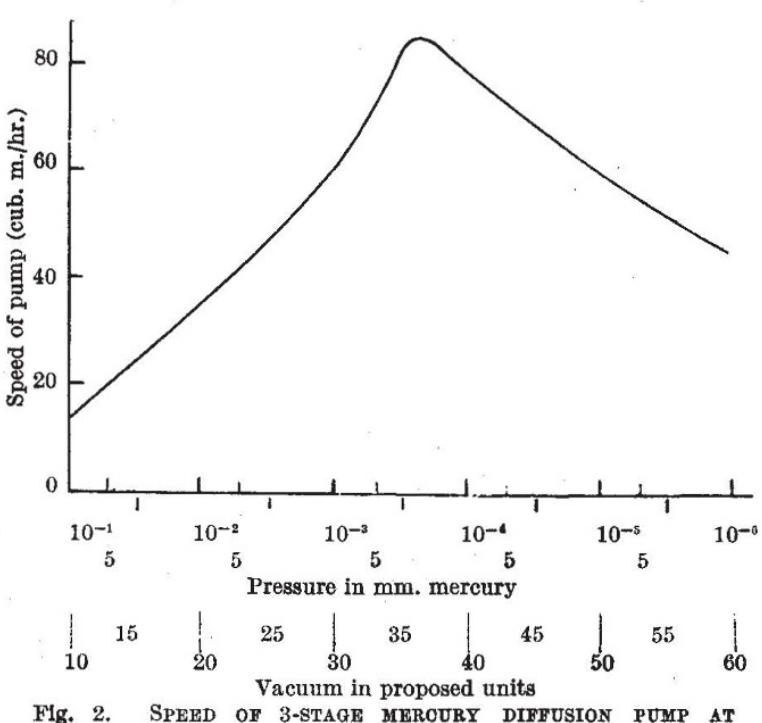

Fig. 2. SPEED OF 3-STAGE MEROURY DIFFUSION PUMP AT

A typical McLeod gauge scale, calibrated in the suggested manner, is shown in Fig. 1, and it may be seen that a more evenly marked scale than usual is obtained. Curves of pump performance, etc., may be plotted on ordinary squared paper, as shown in Fig. 2, and in both cases intermediate values may be more accurately interpolated than by the usual method.

No name has been given to this unit, as it has been found that reference to 'a vacuum of 47 ' is sufficient once the system has been established, but should this suggestion become more widely adopted, a suitable name should be found.

Cathodeon, Ltd.,

Cambridge. Feb. 22.

\section{Reflexion in a Non-Isotropic Medium}

THe way in which the extraordinary ray transmitted by a calcite crystal infringes the laws of simple refraction is, of course, a commonplace and is mentioned in all text-books that deal with polarization.

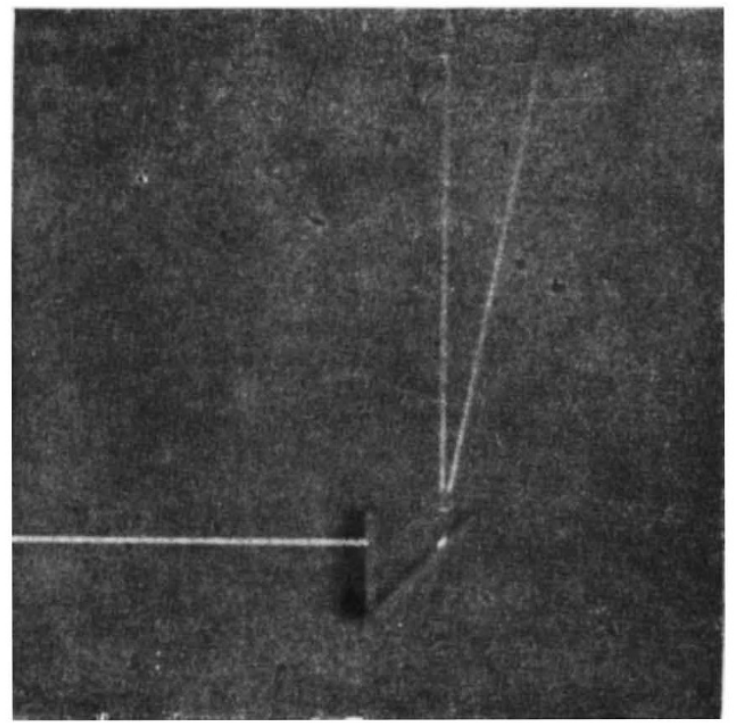

On the other hand, I have not yet found an explicit reference to the infringement of the law of the equality of the angles of incidence and reflexion.

Hence the accompanying photograph (Fig. 1), which illustrates one simple case, may be of interest. It shows the trace, on a sheet of paper, of a ray of unpolarized light incident upon the totally reflecting face of a prism of calcite cut in such a way that the optic axis is parallel to the incident beam. Two plane polarized rays are reflected. The one reflected normally is the ordinary ray, with the electric vector perpendicular to the plane of the paper, whereas the one reflected at an obtuse angle is the extraordinary ray with the electric vector parallel to the plane of the paper. In the latter case it is clear that the angle of incidence made with the totally reflecting surface is not equal to the angle of reflexion.

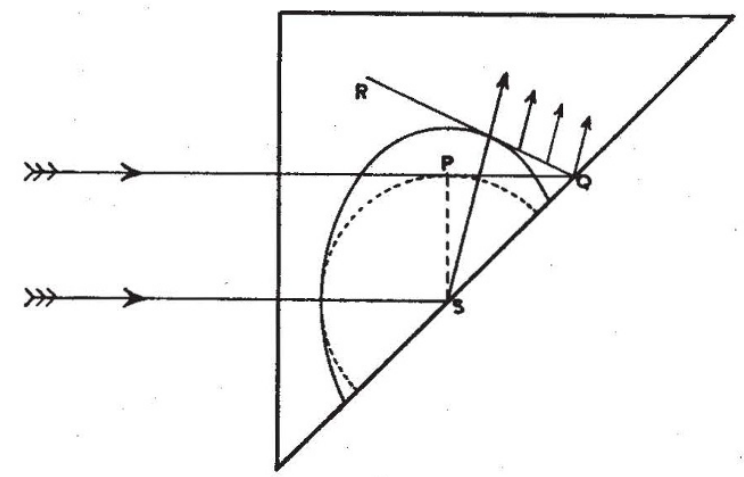

Fig. 2.

The result follows, of course, from the fact that, in the time waves take to travel from $P$ to $Q$ in Fig. 2, the extraordinary wave front that spreads out from $S$ is an ellipse, and the reflected ray is thus propagated in the direction of the arrows on the tangent $Q R$. After refraction this ray gives rise to the emergent ray seen in the photograph. The path of the ray thus calculated (using the accepted values of the ordinary and extraordinary refractive indexes) is in good agreement with the observed path. Gilbert D. West.

Physics Branch,

Military College of Science, Stoke-on-Trent. March 2.

\section{Action of Hydroxylamine on Polysaccharides Oxidized with Periodic Acid}

BARRY ${ }^{1}$ discovered that when a polysaccharido has been oxidized with periodic acid, the oxidation product on warming with phenylhydrazine yields glyoxalosazone. Thus, oxidized starch and cellulose are, theoretically at least, completely broken down into glyoxalosazone and erythrose phenylhydrazone, while 1,3 polysaccharides, such as laminarin, being attacked only at terminal units by periodic acid, have these oxidized terminal units completely removed by phenylhydrazine. It has now been found that a similar reaction takes place with hydroxylamine. Starch oxidized with periodic acid quickly dissolves in boiling water or absolute alcohol on addition of an alcoholic solution of hydroxylamine made by adding alcoholic potash to hydroxylamine 